Ivo VASILJEV

Université Charles, Prague

\title{
LE SUPPLÉTIVISME - PHÉNOMÈNE DU CENTRE OU DE LA PÉRIPHÉRIE ? ÉTUDE TYPOLOGIQUE
}

\section{Phénomène au double caractère marginal et central}

Le supplétivisme ${ }^{1}$ est un sujet qui se trouve tout à fait marginalisé dans la plupart des discours de la linguistique contemporaine, y compris les grammaires des langues flexionnelles, c'est-à-dire des langues où ce phénomène apparaît dans sa forme la plus développée. ${ }^{2}$ D’autre part, tout en étant marginalisé comme objet d'attention des linguistes en général, il semble que le supplétivisme, en tant que phénomène marginal, n’a jamais été considéré comme un sujet digne d'analyse au sein du discours concernant le centre et la périphérie du système de la langue.

On sait, en même temps, que la qualité centrale du type flexionnel, aux yeux de Vladimír Skalička, fondateur de la typologie linguistique structurale, se manifeste par une coexistence parallèle de plusieurs unités sémantiques ou fonctions au sein d'une seule séquence ininterrompue de phonèmes, telles les désinences des noms et des verbes en langues flexionnelles, cf. la forme -us de l'adjectif latin novus, qui indique à la fois le cas nominatif, le nombre singulier et le genre masculin. Ce qu'on appelle le supplétivisme, n'est, en effet, rien d'autre que la même qualité amenée à son apogée, où ce phénomène se répand jusqu'au morphème porteur de la signification lexicale du mot en question, tels les morphes bon-, mel- et opt- des formes des degrés de comparaison bonus, melior et optimus de l'adjectif bonus (bon), dont chacune est composée de deux unités sémantiques, c'est-à-dire de la signification lexicale bon d'un côté et d'une des fonctions grammaticales de degré de comparaison de l'autre. Donc, on peut dire que le supplétivisme est un phénomène caractéristique du type flexionnel tout en étant un phénomène exceptionnel qui n'apparait que dans une partie très limitée du vocabulaire et de la grammaire. Pourtant, il s'agit toujours des mots qui appartiennent au lexique de fréquence très élevée et qui expriment des significations ou des fonctions fondamentales.

Donc, on peut se poser la question de savoir si ce phénomène fait partie du centre du système de la langue, ou, au contraire, de sa périphérie. Nous essayerons, dans cette contribution, de trouver des réponses, en employant la

\footnotetext{
${ }^{1}$ Le terme de supplétivisme est employé ici dans son acception courante, c'est à dire pour désigner les cas où les unités d'un paradigme comportent des bases lexicales différentes (p. ex. je vais - nous $\underline{\text { allons }}$ ). Soulignons que l'approche adoptée dans le présent texte est purement synchronique et typologique. Or, de ce point de vue, le supplétivisme peut se définir comme un phénomène où dans un morphème lexical, un élément sémantique lexical se trouve réuni avec un élément sémantique grammatical bien défini. Ce phénomène ne peut pas y avoir lieu que si un paradigme donné est rempli par des formes à racines différentes comme dans le cas des adjectifs bon/meilleur ou bien/mieux en français.

${ }^{2}$ Un bon exemple est la grammaire académique de la langue tchèque (PETR, 1986), où les diverses manifestations de ce phénomène se trouvent éparpillées dans les chapitres concernant les parties du discours individuelles sans être analysés d'une façon ou de l'autre (PETR, 1986 : 183, 301, 390, 436, 493).
} 
méthode d'analyse typologique. Il se peut que celle-ci nous apporte de nouvelles perspectives permettant une compréhension plus nuancée de la notion même de centre et de la périphérie du système linguistique.

\section{Un territoire inexploré}

Il paraît, d’ailleurs, que le professeur Skalička, qui a classé, sans équivoque et à plusieurs reprises, le supplétivisme parmi les phénomènes caractéristiques du type flexionnel, s'est contenté de constater ce fait sans avoir entrepris l'analyse du phénomène même. La seule exception à ce que nous venons de dire, c'est sa remarque, que le supplétivisme ne se manifeste que dans des mots qui s'emploient fréquemment, ce qui doit être lié au besoin d'assurer le minimum de contraste (SKALIČKA, 2004 : 126). L'explication du phénomène que nous venons de donner ci-dessus et qui a été tirée de la logique générale de l'analyse du type flexionnel effectuée par Vladimír Skalička, revient donc à nous-mêmes.

D’un autre côté, il paraît que Skalička n’a jamais montré, de façon explicite, de l'intérêt vis-à-vis de l'opposition centre/périphérie au sein du système de la langue. Du moins, dans ses oeuvres complètes (SKALIČKA, 2004a, 2004b, 2006) on n'en trouve aucune trace. ${ }^{3}$ Étant donné le grand intérêt que Skalička a manifesté à tous les aspects de la langue et de la parole, ce fait nous frappe comme une chose qui mérite d'être notée. En tout cas, si nous voulons examiner le phénomène des formations supplétives sous la double perspective de la typologie linguistique et de l'opposition du centre et de la périphérie du système de la langue, nous nous trouvons dans un territoire jusqu ici inexploré.

\section{Courte revue de quelques descriptions existantes}

Passons d'abord en revue quelques définitions ou descriptions générales du supplétivisme. On a constaté, que c'est, en principe, «un phénomène d'allomorphie basé sur des éléments qui ne sont pas apparentés du point de vue étymologique »(ČERMÁK, 2001: 192 et 287). Un autre auteur dit que le supplétivisme est l'alternance de deux ou plusieurs morphes lexicaux qui ne sont pas des allomorphes (donc des variantes positionnelles) du même morphème au sein d'un paradigme flexionnel (OSOLSOBĚ, 2002 : 470). D’après MAROUZEAU (1960 : 303), la fonction des formes supplétives est de remplacer les formes absentes dans un paradigme incomplet ou défectif, tandis que le traducteur et rédacteur russes de son dictionnaire préfèrent exprimer la même idée en disant que le supplétivisme consiste dans le remplissage du même paradigme par des formes à racines ou thèmes différents (MAROUZEAU, 1960 : 303). Il est évident que dans toutes ces définitions ou descriptions il ne s’agit que de différentes perspectives qui révèlent tel ou tel aspect du même phénomène. Ce qu'elles ont de commun, c'est que le supplétivisme est toujours encadré dans un paradigme. Nous pouvons encore ajouter que la forme minimum d'un tel paradigme peut être une opposition grammaticale bien définie comme celle de l'aspect perfectif et l'aspect imperfectif des verbes. Sans l'existence de paradigmes et de telles oppositions il ne saurait y

\footnotetext{
${ }^{3}$ On peut constater que même sa contribution au volume consacré spécialement au sujet du centre et de la périphérie du système de la langue (TLP 2) n’a rien à faire avec ce thème.
} 
avoir de supplétivisme. C'est là la condition principale de la possibilité d'un tel phénomène et de son affiliation au type flexionnel dont il est un des phénomènes typiques.

\section{Tableau d'ensemble du phénomène dans une langue flexionnelle}

Il ne sera pas inutile de dresser un tableau d'ensemble du supplétivisme dans une des langues flexionnelles, le tchèque, par exemple, parce que la littérature existante ne se borne, en règle générale, qu'à la citation de quelques exemples.

1) Les paradigmes des pronoms personnels: já (nom. moi) $\mathrm{x}$ mě/mne/mně/mnou (tous les autres cas); ty (nom., voc. toi) x tebe/tě, tobě/ti, tebou (tous les autres cas), on (nom. lui) x jeho/něho, jemu/němu/mu, jeho/ho, něm/ním (tous les autres cas), ona (nom. elle) x ní/jí, ji (tous les autres cas); my (nom. nous) x nás/nám (tous les autres cas), vy (nom. vous) $\mathrm{x}$ vás/vám (tous les autres cas), oni, ony, ona (nom. eux, elles) $\mathrm{x}$ jich/nich/jim/nim/je/ně/jimi/nimi.

2) Noms substantifs : člověk (nom. sg. homme), lidé (nom. pl. hommes) ; Cette opposition se multiplie dans certains noms composés: opočlověk $\mathrm{x}$ opolidé, lidoop x *člověkoop, nadčlověk x nadlidé, pračlověk x pralidé; rok (nom. sg. an, année) x roky, léta (nom. pl.) variantes stylistiques, avec leurs différents paradigmes de déclinaison;

Variantes stylistiques comme: človéčenstvo (archaïque) x lidstvo ;

Adjéctifs dérivatifs : člověčí x lidský, nelidský, nadlidský et des composés qui peuvent, dans quelques cas, varier entre les deux éléments possibles: jednoroční, jednoletý, dvouroční, dvouletý, mais třiletý, čtyřletý, et surtout pétiletý... tisíciletý. Notons que des synonymes n'existent pas au niveau de l'adjectif simple: roční (d'un an), mais letní, comme dérivatif du substantif léto (été, donc estival).

La création de nouvelles appellations étant, en principe, un procédé diachronique, on ne peut pas s'étonner si l'on trouve des noms composés formés quasi-arbitrairement en utilisant, en tant que composant, un élément ou un autre: člověkobijce (à peu prês: misanthrope), lidoop (anthropoïde) lidožrout (cannibale).

3) Paradigmes de comparaison de certains adjectifs et d’adverbes dérivés :

Dobrý (bon) x lepší (meilleur), lépe (mieux), Špatný (mauvais), horší (pire), hůře (pis),

Zlý (méchant) x horší (plus méchant),

Malý (petit) x menši (plus petit), méně (moins),

Velký (grand) x větší (plus grand), více (plus),

4) paradigmes de conjugaison de quelques verbes de base :

být (être) x jsem/jsi/je/jsme/jste/jsou (formes de conjugaison du temps présent x budu/budeš/bude etc. (temps futur) x byl/byla etc. (passé), bud'! (impératif sois!)

mít (avoir) x mám/máš etc. (présent), měl/měla (passé), 
jít (aller) x jdu/jdeš/jde etc. (présent), půjdu etc. (futur), ̌̌ (passé), nechod'! (impératif ne vas pas!)

Le supplétivisme de quelques formes des deux verbes être et aller est encore plus marqué dans la langue parlée :

Bejt x sem, si, sě̌ - être x je suis, tu es) x je (il, elle est) x sme/ste/sou (nous sommes, vous êtes, ils sont),

Jit (aller) x du/dě̌/de etc. (présent), pudu etc. (futur).

5) Opposition d'aspect de quelques verbes fréquents :

Brát ( imparf. prendre) x vzít (perf. prendre), vezmu etc. (futur je prendrai), vzal etc. (passé j'ai pris), vezmi $\mathrm{x}$ neber! (impératif prend-le! $\mathrm{x}$ ne le prend pas !. Dans la langue parlée quelques formes se ressemblent encore moins: vzít $\mathrm{x}$ vemu (je prendrai), vem! x neber! (prend-le! $\mathrm{x}$ ne le prend pas !).

Jit (aller, marcher) $\mathrm{x}$ chodit (aller, marcher) et toutes les formes de conjugaison: jdu (je vais) x chodím (je vais), šel x chodil (il allait).

Klást (imparf. mettre), kladu (je mets) x položit (perf. mettre).

Quoiqu'il ne s'agisse que de trois ou quatre paires de verbes, le supplétivisme se répand sur un vaste champ de verbes dérivés, tels que :

Jit, přejit (traverser), prijit (venir), odejit (partir), vejit (entrer), vyjit (sortir), sejit (descendre), sejit se (se rassembler), zajit (visiter), ujit, najit (trouver), pojit (crêver);

Chodit, prechodit, vychodit, nachodit (accumuler un certain nombre de kilomètres de marche), pochodit (avoir succès); comme les verbes à l'aspect imperfectif deviennent perfectif quand ils prennent un préfixe, leur base subit encore une autre transformation pour renouveler la signification de l'aspect imperfectif :

Přecházet, přicházet, odcházet, vcházet, vycházet $z$, vycházet s, scházet, scházet se, zacházet, ucházet, nacházet, nacházet se, pocházet.

Brát, vybrat, nabrat, sebrat, pribrat, zabrat, ubrat, prebrat, pobrat, Vzít, převzít.

(Le verbe převzít peut entrer en opposition avec deux verbes différents de même signification: prebírat (qui a plusieurs significations) et prejímat (dont la racine est différente tandis que sa signification est exactement identique).

Vybírat, nabírat, sbírat, přibírat, zabírat, ubírat, přebírat

Klást, naklást,

Položit, přeložit, naložit, vyložit, uložit, složit, podložit, odložit, založit, vynaložit.

Pokládat, překládat, nakládat, vykládat, ukládat, skládat, podkládat, odkládat, zakládat.

6) Enfin, il y a quelques formes supplétives qui contribuent à l'opposition des premiers numéraux cardinaux et ordinaux:

Jeden/jedna/jedno (un, une) x první (premier, première),

Dva/dvě (deux) x druhý (deuxième) 
De plus, chaque forme présente son propre éventail de polysémie de sorte que des formes correspondantes peuvent avoir des significations partiellement ou totalement différentes.

Pour compléter le tableau déjà assez compliqué, ajoutons qu'on se sert de ces verbes pour dériver de nouveaux mots. Comme nous l'avons déjà noté, il s'agit là de faits diachroniques, ce qui a pour conséquence qu'il n'y a pas toujours de correspondance précise entre la forme et son contenu. Néanmoins, l'étudiant étranger se pose souvent la question de savoir quelle est la relation entre les deux. Il se trouve fréquemment induit en erreur par la clarté de la forme sur le plan étymologique, tandis que le lien à la vraie signification lexicale du signe est difficile à déterminer. Pour ne citer que quelques exemples, comparons d'abord les deux noms substantifs náklad (charge de marchandise, frais, tirage) et základna (base) x nálož (charge d'explosifs) et záložna (caisse d'épargne), les uns dérivés de la forme imperfective klást, les autres de la forme perfective položit (ou des deux verbes klást et položit), ayant la même signification lexicale (mettre).

\section{Le rôle des perspectives}

On peut donc dire que le phénomène du supplétivisme en langue tchèque est loin d'être marginal. S'il est souvent marginalisé, semble-t-il, par la plupart des linguistes modernes, $c^{\prime}$ est parce que, du point de vue synchronique, il se présente comme un phénomène assez exceptionnel.

Or, il n’apparaît comme tel que dans une description systématique des formes d'une langue en parties du discours quand on tient à souligner les régularités, les prototypes, tout ce qui est productif et « systématique ». Jadis cependant, quand on évaluait le supplétivisme plutôt du point de vue diachronique, de son rôle dans l’histoire et de la généalogie des langues indo-européennes, on était plus conscient de sa valeur: il indiquait clairement l'appartenance de telle ou telle langue à la famille ou à un certain groupe de langues indo-européennes. ${ }^{4}$ De la même façon, il nous semble que l'importance de ce phénomène resurgira dès qu'on commencera à examiner le supplétivisme sous l'angle comparatif ou contrastif surtout si l'on tient compte des exigences de la pédagogie.

Une marginalisation ou amplification de l'importance d'un tel phénomène dépend donc, dans une certaine mesure, du point de vue du chercheur.

\section{L’opposition centre/périphérie, devrait-elle être reconsidérée ?}

$D^{\prime}$ autre part, on doit se poser, de nouveau, la question concernant la nature du système de la langue. On accepte, de nos jours, de ne pas concevoir le système de la langue comme une structure idéale absolument dominée par la régularité et le caractère systématique de ses unités. Une telle conception du système a été réfutée suffisamment et de façon absolument convaincante par František Daneš dans son article bien connu dans le volume des Travaux linguistiques de Prague spécialement dédié aux problèmes du centre et de la périphérie du système de la langue (DANEŠ, 1966) ainsi que par Jiří V. Neustupný (NEUSTUPNÝ, 1961, 1966),

\footnotetext{
${ }^{4}$ Cf. une remarque de N. D. Andreyev et A. A. Reformatskii, les traducteur et rédacteur du Lexique de la terminologie linguistique, p. 303 (MAROUZEAU, 1960).
} 
auquel Daneš se réfère souvent. Selon Daneš, en effet, chaque classe d'unités linguistiques a son centre et sa périphérie, la périphérie étant un champ où on voit certaines unités - membres de cette classe, partiellement dépourvues d'une part des propriétés caractéristiques de la plupart des membres de la classe. Puis il y a des cas de transition qui se dégagent de la périphérie d'une classe pour s'associer à la périphérie, voire au centre même, d’une autre classe (DANEŠ, 1966: 12). Ajoutons, que cette autre classe n'est pas moins centrale dans le système de la langue donnée que la classe d'origine d'où cet élément transitoire s`était dégagé.

On est tout à fait d'accord avec Daneš pour considérer que cette construction « vague » du système de la langue constitue un trait essentiel, constant et universel de ce système. Or, si on confond ce problème de la complexité et de l'asymétrie des classes d'éléments linguistiques avec celui du centre et de la périphérie, cela renforce encore davantage la base sur laquelle un phénomène aussi caractéristique des langues flexionnelles que le supplétivisme, étant déjà exceptionnel et irrégulier dans un système construit comme un ensemble de régularités, peut être analysé comme un phénomène périphérique. Cela nous semble difficile à accepter. D'un autre côté, si le caractère complexe et plein d'irrégularités est vraiment une propriété inhérente et centrale du système de la langue, le terme périphérie utilisé dans ce contexte sera plutôt trompeur.

Donc, nous sommes arrivés à nous poser la question de savoir si une telle conception du centre et de la périphérie est encore utilisable pour le dégagement et la description d'éléments vraiment périphériques, c'est-à-dire faiblement intégrés, non aux classes individuelles d'unités linguistiques, mais au système d'une langue donnée en tant qu'entité. ${ }^{5}$

Pour nous, la notion de périphérie est associée avec celle des périmètres, des lignes frontalières, quoiqu' imaginaires. Le système d'une langue donnée à une époque donnée n'en aura pas moins que trois: une qui le délimite vis-à-vis des innovations au sein de la communauté linguistique, une autre qui sépare le présent et le passé, et enfin et surtout, une qui passe entre la langue donnée et les autres langues, voisines ou non. Certes, les périmètres du système d'une langue sont vagues, perméables et facilement franchissables. Chaque jour on est confronté à de multiples intrusions. Certaines seront rejetées, d'autres acceptées. Nous concevons la périphérie comme une zone grise entre l'acceptation et le rejet de l'innovation quelle que soit son origine d'une part, et entre l'usage commun et la tombée dans l'oubli. L'intégration serait simplement un processus de l'acceptation de l'innovation de plus en plus large par la communauté linguistique. Un tel processus serait bien définissable et, en principe, mesurable.

Selon nous, une telle approche serait plus appropriée, étant donné le caractère extrêmement complexe des systèmes des langues. Ces dernières sont constituées d'unités plus ou moins anciennes, d'origine différente, qui se trouvent reliées par leur fonction au sein du système. Leur caractère systémique et leur valeur résulte

\footnotetext{
${ }^{5}$ L'existence de deux conceptions différentes du centre et de la périphérie n'a jamais été constatée de façon explicite mais elle peut être sous-entendue lorsqu'on compare le titre du volume 2 des TLP (1966) et celui du présent colloque : « du centre et de la périphérie du système de la langue » d’un côté, et «Centre et périphérie dans le système linguistique » de l'autre. En réalité, la seconde conception a déjà été présentée par F. Daně̌, J. Vachek et J. V. Neustupný dans les TLP 2.
} 
de leur interdépendance au sein de ce système, conformément au principe d'opposition posé par Saussure.

\section{Conclusion}

Nous venons de voir que la question que nous nous sommes posée dans le titre de cet article, à savoir si le supplétivisme est un phénomène du centre ou de la péripherie, peut être approchée de deux côtés différents : de celui du supplétivisme et celui de l'opposition centre/périphérie. Tandis que la première approche semble indiquer le caractère central de ce phénomène, la deuxième nous fait croire que la notion même de l'opposition centre/périphérie mérite d'être, une fois de plus, redéfinie.

\section{BIBLIOGRAPHIE}

ČERMÁK, František (2001), Jazyk a jazykověda. Přehled a slovníky. Praha, Karolinum.

MAROUZEAU, J. (1931, 1960), Slovar lingvističeskich terminov. Moskva, Izdatelstvo inostrannoi literatury. [Traduit du: Lexique de la terminologie linguistique, français, allemand, anglais, italien par J. Marouzeau, $3^{\mathrm{e}}$ édition, Paris, 1951].

DANEŠ, František (1966), The Relation of Centre and Periphery as a Language Universal, in TLP 2, Les problèmes du centre et de la périphérie du système de la langue. Prague, Academia, p. 9-21.

NeUSTUPNÝ, Jiří V. (1961), The Assymetry of Phonological Oppositions, in The Bulletin of the Phonetic Society of Japan (Nihon onsei gakkai kaiho), 106, p. $1-6$.

NEUSTUPNÝ, Jiří V. (1966), On the Analysis of Linguistic Vagueness, in TLP 2, Les problèmes du centre et de la périphérie du système de la langue. Prague, Academia, p. 39-51.

OsOLSOBĚ, Klára (2002), Supletivismus, in P. Karlík - M. Nekula - J. Pleskalová (eds), Encyklopedický slovník češtiny. Praha, Lidové noviny, p. 470.

Petr, Jan (éd.), (1986). Mluvnice češtiny (2), Tvarosloví. Praha, Academia.

SKALIČKA, Vladimír (1936) (2004a), Notes sur le redoublement. Sborník Matice Slovenskej 14, p. 19 - 22. Traduction tchèque, in: Vladimír Skalička, Souborné dílo I, p. 123-127.

SKALIČKA, Vladimír (2004a), Souborné dílo, 1. díl (1931 - 1950). Praha, Univerzita Karlova v Praze - Nakladatelství Karolinum.

SKALIČKA, Vladimír (2004b), Souborné dílo, 2. díl (1951 - 1963). Praha, Univerzita Karlova v Praze - Nakladatelství Karolinum.

SKALIČKA, Vladimír (2006), Souborné dílo, 3. díl (1964 - 1994). Praha, Univerzita Karlova v Praze - Nakladatelství Karolinum.

VACHEK, Josef (1966), On the Integration of the Peripheral Elements into the System of Language. In : TLP 2, Les problèmes $d u$ centre et de la périphérie du système de la langue. Prague, Academia, p. 23-37. 


\section{SUMMARY}

While being a subject totally marginalized in most discourses of modern linguistics, suppletion, a supposedly marginal phenomenon, seems to have never been considered a subject worth of analysis within the framework of the centreand-periphery dichotomy. The author argues that from the point of view of language typology as defined by Vladimír Skalička, suppletion can clearly be considered a phenomenon in which inflection reaches its maximum degree, being, therefore, fairly characteristic of languages with a high share in the inflectional type and central to their systems. After reviewing some of the existing definitions of suppletion, the author dresses a general picture of suppletion cases in Czech, one of the typical highly inflectional languages, showing that suppletion in Czech is by no means peripheral. He then suggests that at a point when complexity, vagueness and assymetry of linguistic units are generally understood to be an inherent feature of language systems, the centre-and-periphery dichotomy would be more useful as an analytical tool if reserved for the description and analysis of processes of acceptance by, integration into and/or elimination from the established language system of all kinds of innovations or anachronisms. 